\title{
Age Significantly Affects Response Rate to Outcomes Questionnaires Using Mobile Messaging Software
}

\author{
Toufic R. Jildeh, M.D., Joshua P. Castle, M.D., Muhammad J. Abbas, B.S., \\ Miriam E. Dash, M.S., Noel O. Akioyamen, B.S., and Kelechi R. Okoroha, M.D.
}

\begin{abstract}
Purpose: To investigate the demographic factors that influence time to respond (TTR), time to completion (TTC), and response rate when using a text messaging-based system and to determine the feasibility and applicability of mobile messaging-based services for collection of patient-reported outcomes among orthopaedic sports medicine patients. Methods: On the day of surgery, patient mobile phone number was collected and the automated mobile messaging service (MOSIO, Seattle, WA) messaged patients for 10 "days postoperatively. Patient visual analog scale (VAS) scores were collected 3 times daily, side effects were asked each evening, and Patient-Reported Outcomes Measurement Information System (PROMIS) Pain Interference (PI) Short Form was collected on postoperative day 3 and 7. Results: A total of 177 patients were enrolled in the study. The overall response rate to the survey questions was $75.0 \%$. For all patients, the average TTR of questions was $35.09 \pm 12.57$ minutes. The TTC was $2.75 \pm 3.56$ minutes for PROMIS-PI, 3.51 \pm 1.26 minutes for VAS, and $3.80 \pm 6.87$ for side-effect questions. When patients were stratified into age groups, the youngest group, 16 to 32 years, had the greatest response rate of $85.2 \%$ and patients in the 49 to 59 years group had the lowest response rate of $68.4 \%$ and $69.1 \%$, respectively $(P<.001)$. There was no significant difference in the TTR or TTC for VAS, PROMIS-PI, or side-effect questions when patients were stratified by age or sex groups $(P>.05)$. Conclusions: Collectively, all age groups successfully achieved a mean response rate of $75 \%$; however, significantly lower response rates were observed for patients $>49$ years old. Differences in age and sex did not impact the overall TTR or TTC for VAS, PROMIS-PI, or side-effect questions. Mobile-based applications present as an emerging opportunity to track postoperative outcome scores and reduce clinic survey load. Level of Evidence: Case series, level of evidence IV.
\end{abstract}

$\mathbf{T}$ he importance of value-based care is increasing for physicians, particularly as greater emphasis has been placed on patient-reported outcomes (PROs) by clinicians, insurers, and government-based enterprises. $^{1,2}$ PROs serve as an important surrogate measurement for clinical outcomes and patient satisfaction, especially in the realm of physical, mental, and social

From the Department of Orthopaedic Surgery, Henry Ford Hospital (T.R.J., J.P.C., M.J.A.); Wayne State University School of Medicine (M.E.D., N.O.A.), Detroit, Michigan; and Department of Orthopedic Surgery, Mayo Clinic, Minneapolis, Minnesota (K.R.O.), U.S.A.

The authors report the following potential conflicts of interest or sources of funding: K.R.O. reports other from Stryker, Arthrex, Smith o Nephew, and Zimmer, outside the submitted work. Full ICMJE author disclosure forms are available for this article online, as supplementary material.

Received January 12, 2021; accepted June 28, 2021.

Address correspondence to Toufic R. Jildeh, M.D., Department of Orthopaedic Surgery, 2799 W. Grand Blvd, Detroit, MI 48202. E-mail: touficjildeh@gmail.com

(C) 2021 THE AUTHORS. Published by Elsevier Inc. on behalf of the Arthroscopy Association of North America. This is an open access article under the CC BY-NC-ND license (http://creativecommons.org/licenses/by-nc-nd/4.0/).

2666-061X/2149

https://doi.org/10.1016/j.asmr.2021.06.004 function. ${ }^{2,3}$ Although the use and value of PROs has increased in recent years, there has been a lack of consensus regarding the optimal logistics and use within clinical practice.

In the United States, cell phone ownership among adults has increased from $62 \%$ of the general population in 2002 to $96 \%$ in $2019 .{ }^{4}$ Communicating with patients via mobile devices has become an important modality in health care. It allows for easier communication between providers and patients through appointment and medication reminders, web-based surveys, and medical counseling. ${ }^{5-7}$ In fact, more than one-quarter of community health programs currently use cellphones for health care purposes. ${ }^{8}$ Administering PROs via mobile-based applications has been successful and valid. Investigations into the validity of collecting PROs of orthopaedic patients using an automated mobile messaging based service ${ }^{9}$ have demonstrated a high intraclass correlation between paper and text messaging-based delivery. In addition, it is critical to evaluate not just the validity of mobile messaging-based PROs collection but also the time to response to question and to complete the entire survey collection, as 
Table 1. Demographic Comparison of Patients Based on Age Groups

\begin{tabular}{|c|c|c|c|c|c|}
\hline & $0-32$ years $(\mathrm{N}=79)$ & $33-48$ years $(\mathrm{N}=37)$ & $49-59$ years $(N=39)$ & $60+$ years $(N=22)$ & $P$ Value \\
\hline Procedure & & & & & $<.01$ \\
\hline ACL & $41(52 \%)$ & $14(38 \%)$ & $2(5 \%)$ & $1(5 \%)$ & \\
\hline RCR & $2(3 \%)$ & $6(16 \%)$ & $14(36 \%)$ & $12(55 \%)$ & \\
\hline Meniscus & $16(20 \%)$ & $12(32 \%)$ & $22(56 \%)$ & $9(41 \%)$ & \\
\hline Race & & & & & .01 \\
\hline White & $30(39 \%)$ & $15(42 \%)$ & $25(64 \%)$ & $13(62 \%)$ & \\
\hline Unknown & $15(19 \%)$ & $2(6 \%)$ & $1(3 \%)$ & $1(5 \%)$ & \\
\hline Sex & & & & & .21 \\
\hline Male & $57(72 \%)$ & $20(54 \%)$ & $23(59 \%)$ & $15(68 \%)$ & \\
\hline Female & $22(28 \%)$ & $17(46 \%)$ & $16(41 \%)$ & $7(32 \%)$ & \\
\hline BMI & $27.72(5.92)$ & $28.37(5.22)$ & $30.93(5.88)$ & $29.53(3.40)$ & .03 \\
\hline Presurgery PROMIS-PI & $57.23(7.60)$ & $59.20(5.16)$ & $60.02(6.17)$ & $61.89(5.82)$ & .08 \\
\hline
\end{tabular}

ACL, anterior cruciate ligament; BMI, body mass index; PROMIS-PI, Patient-Reported Outcomes Measurement Information System Pain Interference; RCR, rotator cuff repair.

Bold values are significant at $P<.05$.

shorter survey has been demonstrated to have increased completion rates. ${ }^{10}$

The purposes of this study were to investigate the demographic factors that influence time to respond (TTR), time to completion (TTC), and response rate when using a text messaging-based system and to determine the feasibility and applicability of mobile messaging-based services for collection of PROs among orthopaedic sports medicine patients. We hypothesized that there would be a direct correlation between patient age and the length of time patients take to respond to text messages and complete surveys. We also hypothesized that patients' race, sex, and body mass index (BMI) would not correlate with response time and response rate of patients using a text messaging-based service.

\section{Methods}

The study was reviewed and approved by our institutional review board. Between February 2019 and January 2020, patients who presented to a fellowshiptrained sports surgeon were considered for inclusion (K.R.O). Inclusion criteria included age older than 16 years and the following commonly performed procedures: primary anterior cruciate ligament reconstruction, primary arthroscopic shoulder labrum repair, primary meniscectomy or meniscal repair, and primary rotator cuff repair. Exclusion criteria included nonEnglish-speaking or non-English-reading patients, and patients without access to a cellphone. Nineteen patients declined participation.

During the preoperative consent process, patients were informed that postoperative follow-up information will be collected using automated text messaging service (MOSIO, Seattle WA). On the day of surgery, patient mobile phone was verified for SMS capabilities, and they instructed on how to respond to questions (Appendix Table 1). Their phone number was collected and the automated mobile messaging service (MOSIO) messaged patients for 10 days postoperatively. Patient visual analog scale (VAS) scores were collected 3 times daily ( $9 \mathrm{am}, 1 \mathrm{pm}$, and $7 \mathrm{pm}$ ), side effects were asked each evening at $7 \mathrm{pm}$, and Patient-Reported Outcomes Measurement Information System (PROMIS) Pain Interference (PI) Short Form was collected in the evening on postoperative day 3 and 7 . In addition, each evening at $7 \mathrm{pm}$, patients were asked about how many narcotics they had consumed in the last 24 hours. Both the VAS score and the PROMIS PI form have been previously validated in the literature as metrics for assessing postoperative pain. ${ }^{1,12}$ The mobile-based messaging application sent an automated message to patients at the predefined intervals. The time at which each patient began responding was recorded. The difference between these values was used to calculate TTR for each day. TTC was calculated based on how long it took a patent to complete the entirety of each individual survey. The response rate was defined as the number of responses received divided by the number of survey questions administered over the course of the study period.

The following variables were collected from the patient medical record: demographic data (age, sex, BMI, and race) and workers compensation status. Patients were stratified into cohorts based on age and sex. In accordance with methodology used in previous literature patients were grouped into quartiles based on age (16-32, 33-48, 49-59, and $\geq 60$ years old). ${ }^{13,14}$

\section{Statistical Analysis}

All continuous data were reported as mean \pm standard deviation, whereas categorical data are reported as 
Response Rate

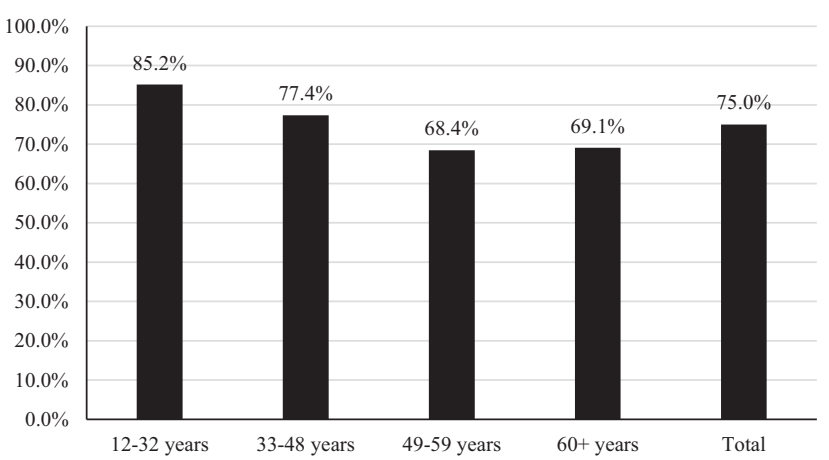

Fig 1. Distribution of response rate based on age grouping.

counts and column percentages, $\mathrm{N}(\%)$. For continuous variables, group comparisons were performed using one-way analysis of variance if the variable was normally distributed and using Kruskal-Wallis tests if the variable was non-normally distributed. Multiple comparisons were performed using a Bonferroni $P$ value. For categorical variables, univariate 2 -group comparisons were performed using $\chi^{2}$ tests when expected cell counts were $>5$ and using Fisher exact tests when expected cell counts were $<5$. Multiple variable regression is performed for each significant outcome and adjusted for each significant demographic variable. Statistical significance was set at $P<.05$. All analyses were performed using SAS 9.4 (SAS Institute Inc., Cary, NC).

\section{Results}

\section{Demographics}

A total of 196 patients were considered for inclusion during the study period. Of these, 177 patients were enrolled in the study. Nineteen patients declined participation due to concerns regarding involvement with any form of research. The overall response rate to the survey questions was $75.0 \%$ (Fig 1). The response rate per postoperative day is demonstrated in Figure 2. The average time from automatic text message to initial response (TTR) was $35.09 \pm 12.57$ minutes. For all patients, the TTC for each question was $2.75 \pm 3.56$ minutes for PROMIS-PI, $3.51 \pm 1.26$ for VAS, and 3.80 \pm 6.87 for side effects. Patient demographics are reported in Table 1. Fifty-eight $(32.8 \%)$ patients underwent anterior cruciate ligament reconstruction, 34 $(19.2 \%)$ rotator cuff repair, 26 (14.7\%) shoulder labrum repair, and 59 (33.3\%) meniscal debridement or repair (Table 1). No significant difference was present in the TTR, TTC, or response rate when patients were stratified by operative procedure $(P>.05)$. When patients were stratified based on age groups, 79 patients were younger than the age of 32 years, 37 patients were age 33 to 48 years, 39 patients were age 49 to 59 years, and 22 patients were older than the age of 60 years. When patients were stratified by sex, there were 116 male patients and 77 female patients.

\section{Response by Age Group}

Patients were stratified into 4 cohorts based on age (Table 1). When we evaluated age groups, reported race $(P=.01)$, BMI $(P=.03)$, and procedure $(P<.01)$ significantly differed between the groups. When we evaluated the cumulative response rate across all postoperative days, the overall response rate to the survey questions was $75.0 \%$ (Fig 1). The youngest group, 16 to 32 years, had the greatest response rate of $85.2 \%$, and patients in the 49 to 59 years' group had the lowest response rate of $68.4 \%$ and $69.1 \%$, respectively $(P<.001)$.

Figure 3 represents the mean TTR to all survey questions for patients in each age group. No significant difference was present between age cohorts. When evaluating the average TTR on each postoperative day, there was no significant difference found between the age groups (Table 2). When we evaluated TTC between patients in each age cohort, there was no significant difference in the mean TTC for VAS scores $(P=.53)$ (Fig 4A), PROMIS-PI questions $(P=.89)$ (Fig 4B), or side-effect questions $(P=.33)$ (Fig $4 \mathrm{C})$.

\section{Response by Sex}

After mean TTR was stratified by sex, overall TTR was not found to significantly differ between sexes (Fig 5). When mean TTR was evaluated on each postoperative day, there was no significant difference found between sexes (Table 3). When we examined the response rate by sex, the total response rate was $79.17 \%$ for male patients and $76.36 \%$ for female patients. When evaluating TTC based on sex, there was no significant difference seen for VAS scores $(P=.11)$ (Fig 6A), PROMIS-PI $(P=.49)$ (Fig 6B), or side-effect questions $(P=.95)($ Fig $6 \mathrm{C})$.

\section{Discussion}

The present study demonstrates that age has a significant effect on the response rate for a text-message

Response Rate Per Day

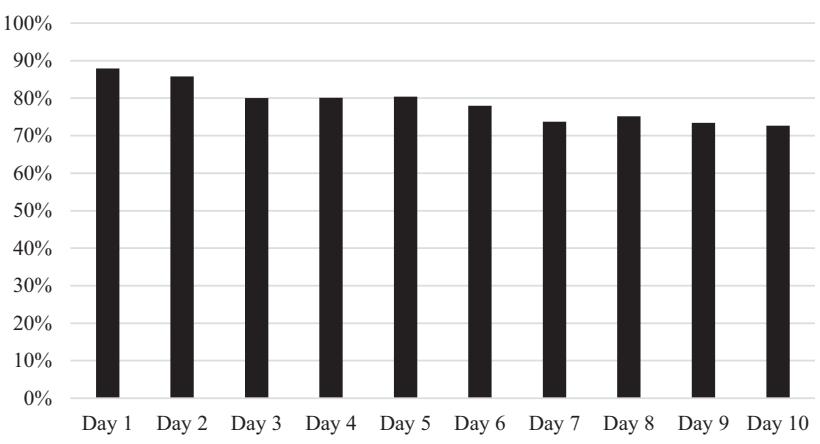

Fig 2. Overall response rate for all patients on each postoperative day. 


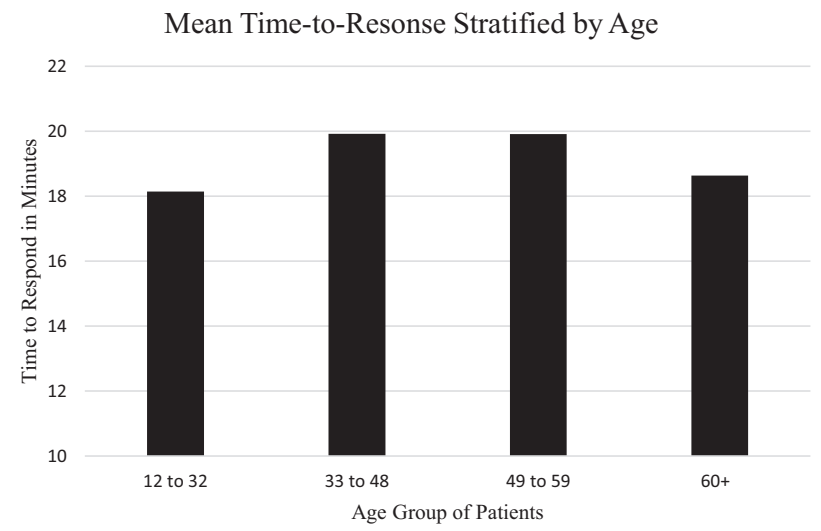

Fig 3. The mean TTR for all patient stratified by age groups. $(P=.94)$. (TTR, time to respond.)

based service collecting postoperative outcomes in patients undergoing routine sports surgery. Collectively, all age groups successfully achieved a mean response rate of $75 \%$; however, significantly lower response rates are observed for patients $>49$ years old. Patient age and sex did not significantly affect TTR or TTC for VAS, PROMIS-PI, or side-effect questions.

A majority of studies reporting data on implementing clinic-based PRO measures and mobile applications for postoperative follow-up have enrolled young patients with an average age less than 60 years old ${ }^{15-20}$; however, there is suggestion that older age may influence response times of patient reported outcomes. In a crosssectional study of 3658 PROMIS forms administered in ambulatory clinics, Kadri et al. ${ }^{14}$ found that patients from the oldest age quartile (mean \pm standard deviation, $70.3 \pm 7.5$ years) had a statistically significant longer TTC as compared with the second quartile (41.2 \pm 4.7 years) $(3.70$ vs 2.87 minutes; $P<.05$ ). They suggested that PROMIS tools can still be used efficiently among older patients; however, they may require more time. Similarly, Morgan et al. ${ }^{21}$ examined the performance of PROMIS Physical Function computer adaptive tests compared with traditionally used PRO measures in patients with proximal humerus fractures. Forty-seven patients, 60 to 88 years old, completed the PROMIS PF survey rather quickly, with a median TTC of 98 seconds (range 45-203 seconds). Beletsky et al. ${ }^{22}$ sought to compare VAS measures of function, strength, and pain against legacy PRO measures in a group of rotator cuff repair patients $55.6 \pm 10.9$ years old. Patients quickly completed surveys with an average TTC of $1.36 \pm 1.12$ minutes for the 3 VAS measures and $1.72 \pm 1.48$ minutes for the PROMIS UE computer adaptive test. These studies together highlight that patients in older age groups are still able to complete surveys rather efficiently. The present study collected PRO measures using a mobile text message-based application. We found that the average time for patients to begin responding to questions after the first message was sent was $35.09 \pm 12.57$ minutes, and the average TTC for PROMIS-PI, VAS, and side-effect questions was unaffected by age. While we cannot directly compare TTC times with these aforementioned studies due to different pathologies studied and survey tools used, our results suggest that mobile-based outcome collection efficiently gathers data without differences in completion time in older patients. The average TTR was unaffected by age and showed that patients took just more than a half hour (average 35 minutes) from text message receipt to responding. These results in combination emphasize that that mobile-based collection tools can perform efficiently in terms of TTC and TTR regardless of age.

Conflicting evidence exists on the effect of age on response rate with a mobile application. Premkumar et al. implemented a text message-based platform to track pain and opioid use for 6 weeks after common orthopaedic procedures for patients on average $59.4 \pm$ 10.9 years old. ${ }^{23}$ Their group reported an excellent response rate of $96.1 \%$ (173 of 180 participants) with further logistic regression showing that age was not associated with nonresponse. However, in their study, adequate response was defined as answering greater than $50 \%$ of text messages received. This threshold of $50 \%$ may be too low to capture key differences in response rates between groups of patients. In contrast, Geerds et al. ${ }^{24}$ examined the use of a mobile application for monitoring outcomes after hip fractures. Patients in this study were on average 80.5 years old. Only $26.4 \%$ of participants downloaded the application and $3.4 \%$ of those patients completed the questionnaire within 12 to 18 weeks after the operation. Reasons for not completing the questionnaire included technical problems with the app, cognitive disorders, patient dependency on caregivers, and forgetting about the study after discharge. Despite high levels of smartphone expertise and self-reported intention to use the app, the application was not successfully implemented in this elderly age group. Although our cohort of interest differed in pathology and follow-up length, our findings

Table 2. Average TTR in Minutes Per Day for Age Cohorts

\begin{tabular}{lcccrc}
\hline & 16 to 32 & 33 to 48 & 49 to 59 & $60+$ & $P$ Value \\
\hline Day 1 & 21.38 & 13.82 & 19.44 & 23.97 & .8 \\
Day 2 & 22.15 & 20.95 & 24.43 & 15.35 & .8 \\
Day 3 & 11.04 & 11.89 & 12.00 & 9.59 & .76 \\
Day 4 & 17.63 & 32.80 & 19.62 & 32.03 & .81 \\
Day 5 & 20.76 & 24.65 & 28.91 & 27.10 & .95 \\
Day 6 & 26.76 & 22.27 & 28.30 & 16.89 & .69 \\
Day 7 & 12.15 & 14.67 & 11.07 & 12.18 & .98 \\
Day 8 & 22.14 & 23.33 & 14.16 & 30.31 & .63 \\
Day 9 & 26.00 & 25.15 & 26.87 & 17.01 & .87 \\
Day 10 & 22.71 & 26.06 & 29.15 & 20.53 & .94 \\
\hline
\end{tabular}

TTR, time to respond. 


\section{A Mean TTC for VAS Score Questions}

Fig 4. (A) The mean time to respond in minutes for all VAS questions when stratified by age group. (B) The mean time to respond in minutes for all PROMIS-PI questions when stratified based on age group. (C) The mean time to respond in minutes for all side effect questions when stratified based on group. (PROMIS-PI, PatientReported Outcomes Measurement Information System Pain Interference; TTC, time to completion; VAS, visual analog scale.)

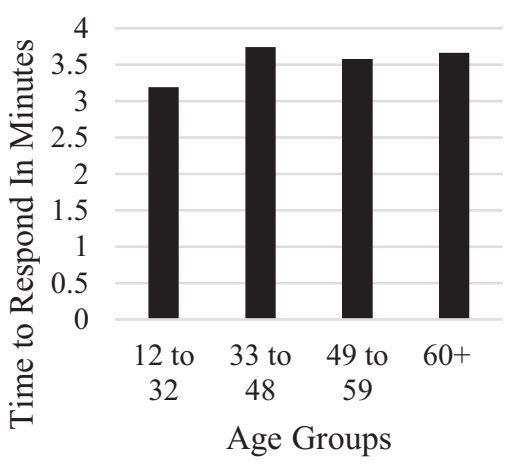

\section{B Mean TTC for PROMIS-PI}

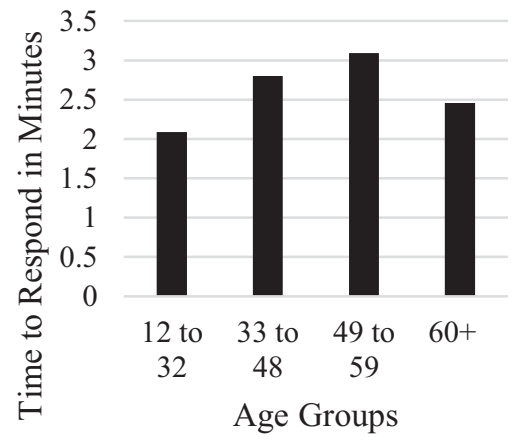

\section{Mean TTC for Side Effect Questions}

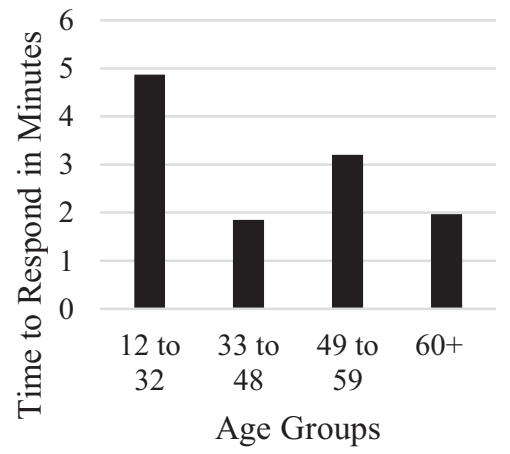

similarly suggest that increasing age hinders response rate within 2 weeks after surgery, with patients $49+$ years old have significantly lower response rates compared with the youngest group, 16 to 32 years old $(85.2 \%$ vs $68.4 \%, P \leq .001)$. Our findings, which specifically focused on sports procedures, are in contrast to the results of Premkumar et al. ${ }^{23}$ who studied a wide variety of pathologies. Although studies have shown that patients $>55$ years old are willing to use mobile applications for postoperative follow-up, ${ }^{25,26}$ there is still a barrier in response rate with application use in older age groups. As suggested by Geerds et al., ${ }^{24}$ older age groups may require further education on the importance of the application. Nonetheless, our findings demonstrate that the majority of patients were compliant with the application, with an average response rate of $75 \%$, but further attention to these older age groups is warranted to improve their compliance.

The effect of sex on performance of PROs has been reported in the literature. Teresi et al. ${ }^{27}$ sought to examine performance of PROMIS PI items based on demographic factors in a cancer and palliative care populations. Differential item functioning or item bias refers to participants with the same underlying trait (such as a specific pain level) will have different probabilities of reporting a response, suggesting that a

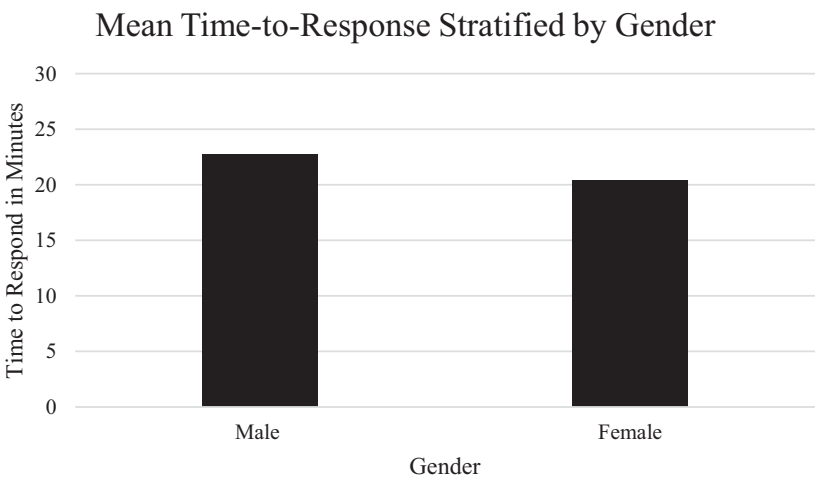

Fig 5. The mean TTR for all patient stratified by sex $(P=.50)$ (TTR, time to respond.) 
Table 3. Average TTR Average TTR in Minutes Per Day for Sex Cohorts

\begin{tabular}{lllc}
\hline & Male & Female & $P$ Value \\
\hline Day 1 & 22.03 & 22.83 & .94 \\
Day 2 & 33.29 & 19.18 & .23 \\
Day 3 & 11.88 & 10.29 & .81 \\
Day 4 & 38.82 & 35.38 & .86 \\
Day 5 & 27.88 & 32.3 & .74 \\
Day 6 & 28.43 & 29.06 & .96 \\
Day 7 & 14.79 & 11.33 & .66 \\
Day 8 & 27.85 & 25.54 & .87 \\
Day 9 & 31.18 & 27.14 & .78 \\
Day 10 & 31.58 & 26.79 & .74 \\
\hline
\end{tabular}

TTR, time to respond.

participants membership in a group or demographic may influence how they respond to a question. Their analysis found no difference in terms of sex. Differential item functioning also was observed for PROMIS Physical Function, PI, and Pain Behavior in patients with osteoarthritis, rheumatoid arthritis, or undergoing physical therapy for a musculoskeletal condition. ${ }^{28}$ However, the magnitude and impact of these items were also negligible. Furthermore, Kadri et al. ${ }^{14}$ further explored the role of sex by assessing factors impacting TTC of PROMIS scores for common arthroscopic surgeries. The authors performed their study with surveys delivered in the clinic setting rather than at home from a mobile device. They found no significant difference in TTC between male and female participants. In line with previous literature, our study did not show differential effects TTR or TTC based on sex. Response times to PROMIS-PI, VAS, and side-effect questions remained prompt regardless of sex, race, or ethnicity. In sum, these findings suggest that PROMIS tools and questionnaires perform reliably and efficiently despite racial or ethnic differences, especially in a mobile based application.

\section{Limitations}

This study was not without limitations. The follow-up in the study was limited to 14 days, thus preventing us from making conclusions beyond this timeframe and impacting the generalizability to other investigations that last longer. In addition, factors linked with poor compliance such as cognitive disabilities, technologic literacy, patient-perceived usability, and satisfaction were outside the scope of this study. There is also potential for observational bias, as patients were made aware of study participation; however, in an effort to reduce potential bias, patients were not aware that the response rate, TTR, and TTC were being recorded. In

\section{A Mean TTC for VAS Score Questions}

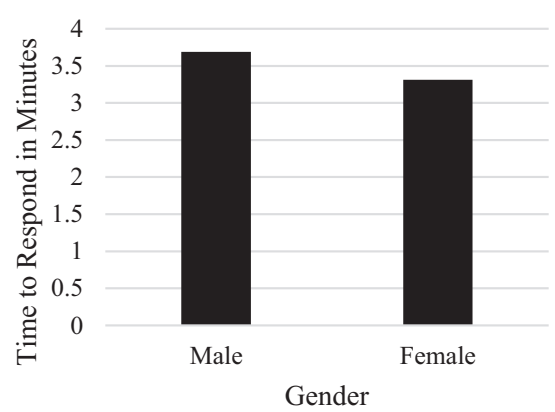

C

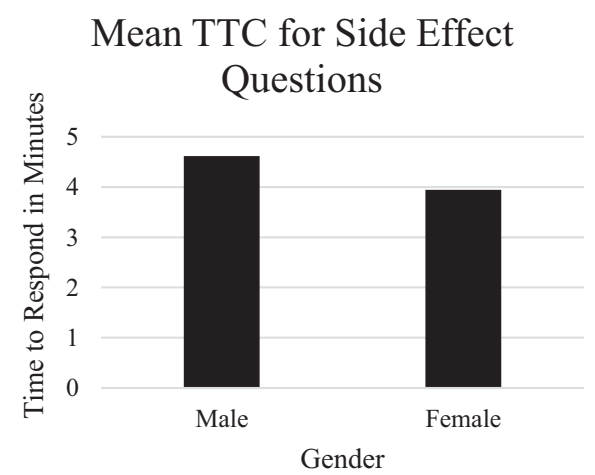

B Mean TTC for PROMIS-PI Questions

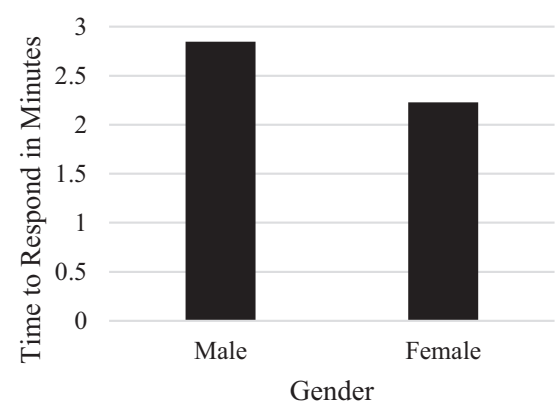

Fig 6. (A) The mean time-toresponse in minutes for all VAS questions when stratified by sex $(P=.12)$. (B) The mean time to respond in minutes for all PROMIS-PI questions when stratified by sex $(P=.49)$. (C) The mean time-to-response in minutes for all side- effect questions when stratified by sex $(P=.95)$. (PROMIS-PI, PatientReported Outcomes Measurement Information System Pain Interference; TTC, time to completion; VAS, visual analog scale.) 
addition, heterogenicity in operative intervention exists within the observed cohort. This variability may lead to additional bias in response rate as rotator cuff repair patients are more likely to be older and have reduced upper-extremity function that may limit mobile device usage. Future studies should assess for barriers to using the text message application with patient surveys after the completion of the study and should evaluate for mobile device use competency preoperatively.

\section{Conclusions}

Collectively, all age groups successfully achieved a mean response rate of $75 \%$; however, significantly lower response rates are observed for patients $>49$ years old. Differences in age and sex did not impact the overall TTR or TTC for VAS, PROMIS-PI, or side-effect questions. Mobile-based applications present as an emerging opportunity to track postoperative outcome scores and reduce clinic survey load.

\section{References}

1. Anker SD, Agewall S, Borggrefe M, et al. The importance of patient-reported outcomes: A call for their comprehensive integration in cardiovascular clinical trials. Eur Heart J 2014;35:2001-2009.

2. Deshpande PR, Rajan S, Sudeepthi BL, Abdul Nazir CP. Patient-reported outcomes: A new era in clinical research. Perspect Clin Res 2011 ;2:137-144.

3. Mercieca-Bebber R, King MT, Calvert MJ, Stockler MR, Friedlander M. The importance of patient-reported outcomes in clinical trials and strategies for future optimization. Patient Relat Outcome Meas 2018;9:353-367.

4. Mobile Fact Sheet. Vol 2020. Internet $\&$ Technology: Pew Research Center; 2019. https://www.pewresearch.org/ internet/fact-sheet/mobile/. Accessed December 20, 2020.

5. Benhamou PY, Melki V, Boizel R, et al. One-year efficacy and safety of Web-based follow-up using cellular phone in type 1 diabetic patients under insulin pump therapy: The PumpNet study. Diabetes Metab 2007;33:220-226.

6. Kinnula S, Renko M, Tapiainen T, Pokka T, Uhari M. Postdischarge follow-up of hospital-associated infections in paediatric patients with conventional questionnaires and electronic surveillance. J Hosp Infect 2012;80:13-16.

7. Lau C, Churchill RS, Kim J, Matsen FA 3rd, Kim Y. Asynchronous web-based patient-centered home telemedicine system. IEEE Trans Biomed Eng 2002;49: 1452-1462.

8. Lee JH. Future of the smartphone for patients and healthcare providers. Healthc Inform Res 2016;22:1-2.

9. Anthony CA, Lawler EA, Glass NA, McDonald K, Shah AS. Delivery of patient-reported outcome instruments by automated mobile phone text messaging. Hand (N Y) 2017;12:614-621.

10. Kost RG, de Rosa JC. Impact of survey length and compensation on validity, reliability, and sample characteristics for ultrashort-, short-, and long-research participant perception surveys. J Clin Transl Sci 2018;2:31-37.
11. Price DD, McGrath PA, Rafii A, Buckingham B. The validation of visual analogue scales as ratio scale measures for chronic and experimental pain. Pain 1983;17:45-56.

12. Dvorzhinskiy A, Gausden EB, Levack AE, et al. The performance of PROMIS computer adaptive testing for patient-reported outcomes in hip fracture surgery: A pilot study [published online January 2, 2021]. Arch Orthop Trauma Surg. https://doi.org/10.1007/s00402-02003640-y.

13. Lizzio VA, Blanchett J, Borowsky P, et al. Feasibility of PROMIS CAT administration in the ambulatory sports medicine clinic with respect to cost and patient compliance: A single-surgeon experience. Orthop J Sports Med 2019;7:2325967118821875.

14. Kadri O, Jildeh TR, Meldau JE, et al. How long does it take for patients to complete PROMIS scores? An assessment of PROMIS CAT questionnaires administered at an ambulatory sports medicine clinic. Orthop J Sports Med 2018;6: 2325967118791180.

15. Higgins J, Chang J, Hoit G, Chahal J, Dwyer T, Theodoropoulos J. Conventional follow-up versus mobile application home monitoring for postoperative anterior cruciate ligament reconstruction patients: A randomized controlled trial. Arthroscopy 2020;36: 1906-1916.

16. Semple JL, Sharpe S, Murnaghan ML, Theodoropoulos J, Metcalfe KA. Using a mobile app for monitoring postoperative quality of recovery of patients at home: A feasibility study. JMIR Mhealth Whealth 2015;3:e18.

17. Lu K, Marino NE, Russell D, et al. Use of short message service and smartphone applications in the management of surgical patients: A systematic review. Telemed $J E$ Health 2018;24:406-414.

18. Lu Y, Beletsky A, Nwachukwu BU, et al. Performance of PROMIS physical function, pain interference, and depression computer adaptive tests instruments in patients undergoing meniscal surgery. Arthrosc Sports Med Rehabil 2020;2:e451-e459.

19. Hung M, Stuart AR, Higgins TF, Saltzman CL, Kubiak EN. computerized Adaptive Testing Using the PROMIS physical function item bank reduces test burden with less ceiling effects compared with the short musculoskeletal function assessment in orthopaedic trauma patients. J Orthop Trauma 2014;28:439-443.

20. Bernstein DN, Houck JR, Hammert WC. A Comparison of PROMIS UE versus PF: Correlation to PROMIS PI and depression, ceiling and floor effects, and time to completion. J Hand Surg Am 2019;44:901.e901-901.e907.

21. Morgan JH, Kallen MA, Okike K, Lee OC, Vrahas MS. PROMIS physical function computer adaptive test compared with other upper extremity outcome measures in the evaluation of proximal humerus fractures in patients older than 60 years. J Orthop Trauma 2015;29: 257-263.

22. Beletsky A, Nwachukwu BU, Gorodischer T, et al. Psychometric properties of visual analog scale assessments for function, pain, and strength compared with diseasespecific upper extremity outcome measures in rotator cuff repair. JSES Int 2020;4:619-624.

23. Premkumar A, Lovecchio FC, Stepan JG, et al. A novel mobile phone text messaging platform improves collection 
of patient-reported post-operative pain and opioid use following orthopedic surgery. HSS J 2019;15:37-41.

24. Geerds MAJ, Nijmeijer WS, Hegeman JH, VollenbroekHutten MMR. Mobile app for monitoring 3-month postoperative functional outcome after hip fracture: Usability study. JMIR Hum Factors 2020;7:e16989.

25. Abelson JS, Symer M, Peters A, Charlson M, Yeo H. Mobile health apps and recovery after surgery: What are patients willing to do? Am J Surg 2017;214:616-622.

26. Scherer J, Keller F, Pape HC, Osterhoff G. Would patients undergo postoperative follow-up by using a smartphone application? BMC Surg 2020;20:229.
27. Teresi JA, Ocepek-Welikson K, Cook KF, et al. Measurement equivalence of the Patient Reported Outcomes Measurement Information System $((\mathrm{R}))$ (PROMIS $((\mathrm{R}))$ ) Pain Interference Short Form Items: Application to ethnically diverse cancer and palliative care populations. Psychol Test Assess Model 2016;58:309-352.

28. Crins MHP, Terwee CB, Ogreden O, et al. Differential item functioning of the PROMIS physical function, pain interference, and pain behavior item banks across patients with different musculoskeletal disorders and persons from the general population. Qual Life Res 2019;28: $1231-1243$. 


\section{Appendix Table 1. Text Messaging-Based Questionnaire}

Time: Postoperative day 1 (morning)

Question: What is your pain right now? $(0-10)$

Time: Postoperative day 1 (afternoon)

Question: What is your pain right now? $(0-10)$

Time: Postoperative day l (evening)

Question: What is your pain right now? (0-10)

Question: How many oxycodone or Norco pills have you taken since the morning today?

Question: Which of the following side effects have you experienced: constipation, nausea, diarrhea, upset stomach, drowsiness, loopiness, none?

Time: Postoperative day 2 (morning)

Question: What is your pain right now? (0-10)

Time: Postoperative day 2 (afternoon)

Question: What is your pain right now? (0-10)

Time: Postoperative day 2 (evening)

Question: What is your pain right now? (0-10)

Question: How many oxycodone or Norco pills have you taken since the morning today?

Question: Which of the following side effects have you experienced: constipation, nausea, diarrhea, upset stomach, drowsiness, loopiness, none

Time: Postoperative day 3 (morning)

Question: What is your pain right now? $(0-10)$

Time: Postoperative day 3 (afternoon)

Question: What is your pain right now? $(0-10)$

Time: Postoperative day 3 (evening)

Question: What is your pain right now? (0-10)

Question: How many oxycodone or Norco pills have you taken since the morning today?

Question: Which of the following side effects have you experienced: constipation, nausea, diarrhea, upset stomach, drowsiness, loopiness, none

Question: How much is the pain interfering with your enjoyment of life $(1-5 ; 1=$ not at all, $5=$ very much)?

Question: How much is the pain interfering with your ability to concentrate? $(1-5 ; 1=$ not at all, $5=$ very much)?

Question: How much is the pain interfering with your day to day activities? $(1-5 ; 1=$ not at all, $5=$ very much)?

Question: How much is your pain interfering with your enjoyment of recreation activities? $(1-5 ; 1=$ not at all, 5 = very much)?

Question: How much is your pain interfering with your tasks away from home (e.g., running errands)? (1$5 ; 1=$ not at all, $5=$ very much)?

Question: In the past 7 days, how often is the pain keeping your from socializing with others? $(1-5 ; 1=$ never, 5 = always)?

Time: Postoperative day 4 (morning)

Question: What is your pain right now? (0-10)

Time: Postoperative day 4 (afternoon)
Question: What is your pain right now? (0-10)

Time: Postoperative day 4 (evening)

Question: What is your pain right now? (0-10)

Question: How many oxycodone or Norco pills have you taken since the morning today?

Question: Which of the following side effects have you experienced: constipation, nausea, diarrhea, upset stomach, drowsiness, loopiness, none

Time: Postoperative day 5 (morning)

Question: What is your pain right now? $(0-10)$

Time: Postoperative day 5 (afternoon)

Question: What is your pain right now? $(0-10)$

Time: Postoperative day 5 (evening)

Question: What is your pain right now? $(0-10)$

Question: How many oxycodone or Norco pills have you taken since the morning today?

Question: Which of the following side effects have you experienced: constipation, nausea, diarrhea, upset stomach, drowsiness, loopiness, none

Time: Postoperative day 6 (morning)

Question: What is your pain right now? (0-10)

Time: Postoperative day 6 (afternoon)

Question: What is your pain right now? (0-10)

Time: Postoperative day 6 (evening)

Question: What is your pain right now? (0-10)

Question: How many oxycodone or Norco pills have you taken since the morning today?

Question: Which of the following side effects have you experienced: constipation, nausea, diarrhea, upset stomach, drowsiness, loopiness, none

Time: Postoperative day 7 (morning)

Question: What is your pain right now? (0-10)

Time: Postoperative day 7 (afternoon)

Question: What is your pain right now? (0-10)

Time: Postoperative day 7 (evening)

Question: What is your pain right now? (0-10)

Question: How many oxycodone or Norco pills have you taken since the morning today?

Question: Which of the following side effects have you experienced: constipation, nausea, diarrhea, upset stomach, drowsiness, loopiness, none

Question: How much is the pain interfering with your enjoyment of life $(1-5 ; 1=$ not at all, $5=$ very much)?

Question: How much is the pain interfering with your ability to concentrate? $(1-5 ; 1=$ not at all, $5=$ very much)?

Question: How much is the pain interfering with your day to day activities? $(1-5 ; 1=$ not at all, $5=$ very much)?

Question: How much is your pain interfering with your enjoyment of recreation activities? $(1-5 ; 1=$ not at all, 5 = very much)?

Question: How much is your pain interfering with your tasks away from home (e.g., running errands)? (1$5 ; 1=$ not at all, $5=$ very much)? 
Question: In the past 7 days, how often is the pain keeping your from socializing with others? $(1-5 ; 1=$ never, 5 = always)?

Time: Postoperative day 8 (morning)

Question: What is your pain right now? (0-10)

Time: Postoperative day 8 (afternoon)

Question: What is your pain right now? (0-10)

Time: Postoperative day 8 (evening)

Question: What is your pain right now? (0-10)

Question: How many oxycodone or Norco pills have you taken since the morning today?

Question: Which of the following side effects have you experienced: constipation, nausea, diarrhea, upset stomach, drowsiness, loopiness, none

Time: Postoperative day 9 (morning)

Question: What is your pain right now? $(0-10)$

Time: Postoperative day 9 (afternoon)

Question: What is your pain right now? (0-10)
Time: Postoperative day 9 (evening)

Question: What is your pain right now? (0-10)

Question: How many oxycodone or Norco pills have you taken since the morning today?

Question: Which of the following side effects have you experienced: constipation, nausea, diarrhea, upset stomach, drowsiness, loopiness, none

Time: Postoperative day 10 (morning)

Question: What is your pain right now? (0-10)

Time: Postoperative day 10 (afternoon)

Question: What is your pain right now? (0-10)

Time: Postoperative day 10 (evening)

Question: What is your pain right now? $(0-10)$

Question: How many oxycodone or Norco pills have you taken since the morning today?

Question: Which of the following side effects have you experienced: constipation, nausea, diarrhea, upset stomach, drowsiness, loopiness, none 